\title{
1. AOTD-Seminar in Fulda
}

Martin Hessmann, Christian Heiß

Am 24. August 2018 fand in Fulda das erste Gießener-Fuldaer AOTrauma-Symposium unter der Leitung von Prof. Dr. Martin Hessmann und Prof. Dr. Christian Heiß statt. Die wissenschaftliche Veranstaltung befasste sich schwerpunktmäßig mit Kontroversen in der Frakturbehandlung an der unteren Extremität. Insgesamt gliederte sich das mit 57 Teilnehmenden und 17 Referenten sehr gut besuchte Symposium in 4 Themenblöcke rund um das proximale und distale Femur sowie rund um die proximale Tibia und den distalen Unterschenkel mit Fuß.

Am proximalen Femur wurden die verschiedenen operativen Therapieoptionen einander gegenübergestellt. Kritisch beleuchtet wurden die Vorteile und Grenzen der gelenkerhaltenden Osteosynthese bei der medialen Schenkelhalsfraktur. Einig waren sich die Experten, dass winkelstabile Implantate mechanische und funktionelle Vorteile aufweisen. In Bezug auf die endoprothetische Versorgung wurden die Vor- und Nachteile der Hemi- und Totalendoprothetik kontrovers diskutiert. Beide Verfahren sind etabliert. Eine eindeutige Überlegenheit einer der beiden Prothesenvarianten ist bis heute nicht eindeutig bewiesen. Bei der Wahl des Implantates sind Patientenfaktoren und Lebenserwartung ausschlaggebend. Die

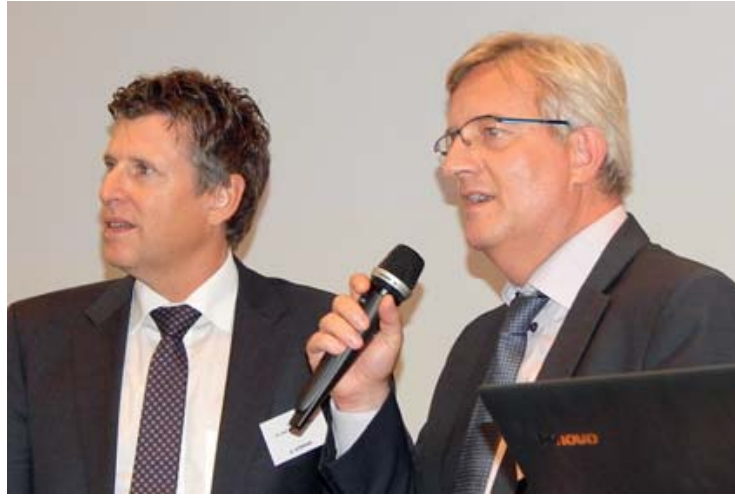

- Abb. 1 Die Leiter Martin Hessmann und Christian Heiß. Quelle: AO Foundation

Rolle der zementfreien Prothese, insbesondere in der Kurzschaftvariante, wurde weiterhin kritisch gesehen und sollte einem ausgewählten Krankengut vorbehalten bleiben.

Bei der per- und intertrochantären Fraktur haben sowohl die intra- als auch die extramedulläre Osteosynthese ihre

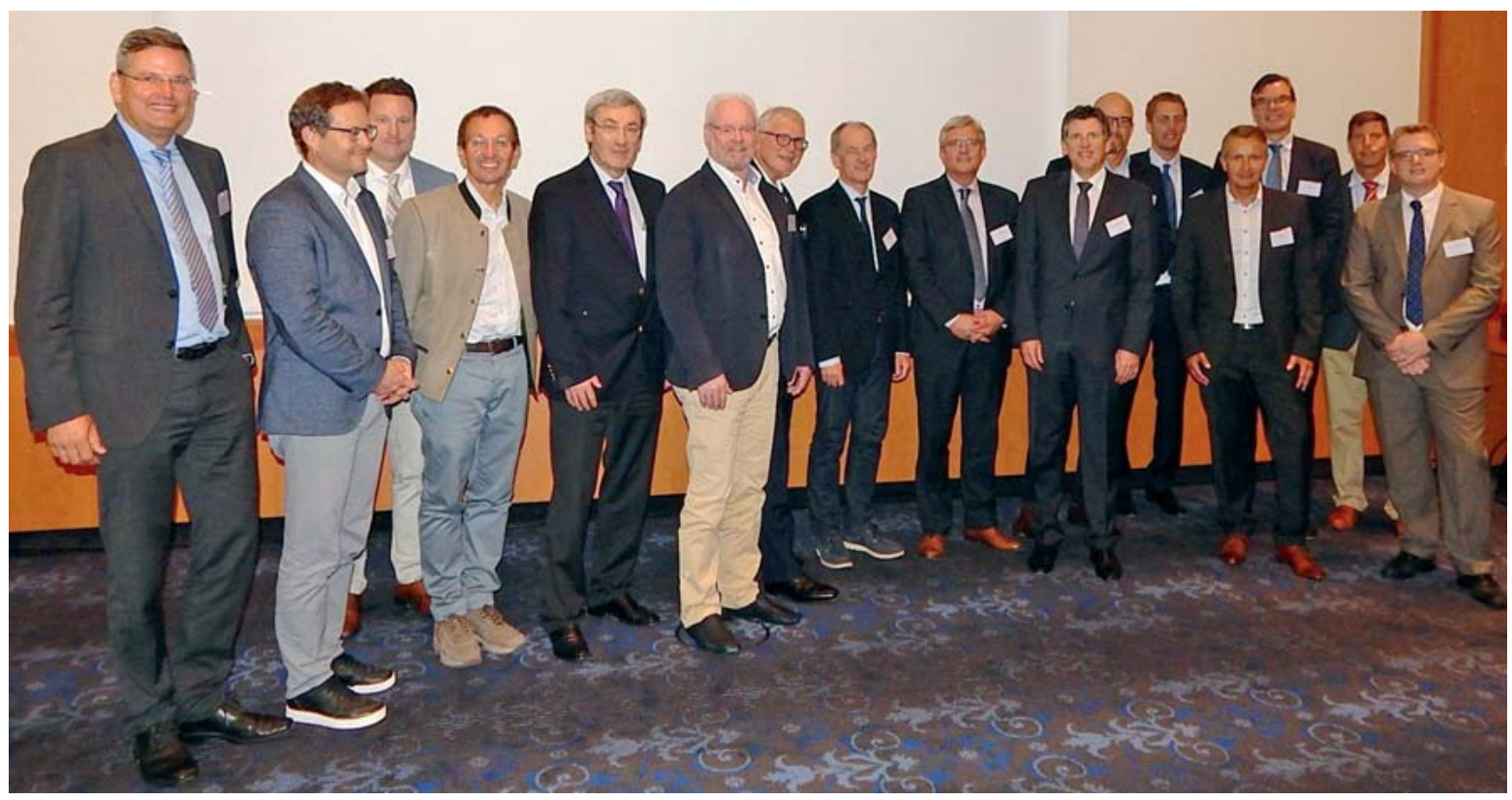

- Abb. 2 Die Referenten des Seminars. Quelle: AO Foundation 
Berechtigung. Die DHS-Osteosynthese mit oder ohne Trochanterabstützplatte stellt insbesondere bei den stabilen A1- und A2-Frakturen nach wie vor eine gute und kostengünstige Behandlungsalternative zur Nagelung dar. Im per- und subtrochantären Bereich sind die Qualität der Reposition und die Implantatlage für das Ergebnis entscheidend. Die gedeckt-offene Reposition ist daher bei geschlossen nicht anatomisch reponierbaren Frakturen Methode der Wahl. Sparsam als Repositions- und Fixationshilfe eingesetzte Cerclagedrähte haben bei grob dislozierten Frakturen ihre Berechtigung.

Bei den periprothetischen Frakturen ist die Therapiewahl, Osteosynthese oder Prothesenwechsel, nicht nur von der Frakturmorphologie und -lokalisation abhängig, sondern auch von der Anamnese (vorbestehende Schmerzen) und vom einliegenden Prothesentyp. Analyse der Röntgenvoraufnahmen sowie die ergänzende CT-Diagnostik wurden einhellig als wichtige Entscheidungshilfen bei der Therapiewahl gesehen.

Am distalen Femur kommen sowohl die winkelstabile Platte als auch der retrograde Nagel zum Einsatz. Da viele distale Femurfrakturen heute bei osteoporotischen Knochenverhältnissen auftreten, haben auch kombinierte Verfahren aus Stabilitätsgründen ihre Berechtigung. Kombinationsosteosynthesen werden daher zunehmend häufiger eingesetzt.

Bei den Tibiakopffrakturen sowie bei den metaphysären proximalen Tibiafrakturen sind die Frakturmorphologie und die Knochenqualität für die chirurgische Strategie ausschlaggebend. Zunehmend werden Doppelplattenosteosynthesen in unterschiedlichen Konfigurationen vorgenommen. Mediale, laterale und dorsale Zugänge sollten daher heute zum Standardrepertoire des Chirurgen gehören. Moderne Marknägel mit stabilen 3-D-Verriegelungsmöglichkeiten sind heute bei den extraartikulären Frakturen eine Alternative zur winkelstabilen eingeschobenen Platte. Neue, insbesondere suprapatellare Zugangswege erleichtern die Reposition und Nagelinsertion und werden daher zunehmend verwendet, auch weil eindeutige Nachteile des transartikulären Zugangs für das Kniegelenk bisher nicht nachgewiesen werden konnten. Allerdings ist eine kritische Beobachtung möglicher Nachteile weiterhin erforderlich.

Der letzte Teil des Symposiums befasste sich mit Verletzungen des Pilons und des Fußes. Ähnlich wie an der proximalen Tibia sollte der Chirurg heute mit den unterschiedlichen Zugängen zur distalen Tibia vertraut sein. Bei kritischen Weichteilverhältnissen stellt die intramedulläre Osteosynthese bei den Frakturen mit einfacher Gelenkkomponente eine gute Behandlungsalternative dar. Allerdings erfordert die Technik eine große Erfahrung in der Marknagelung. Eine komplett neue, aber interessante Technik stellt die retrograde Tibianagelung dar,

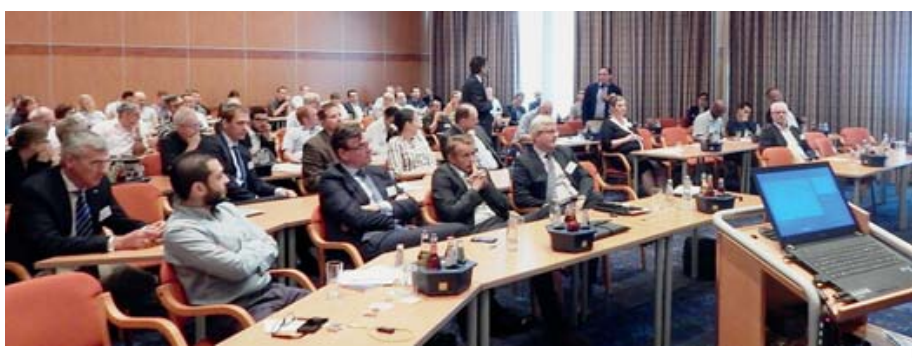

- Abb. 3 Hörsaal. Quelle: AO Foundation

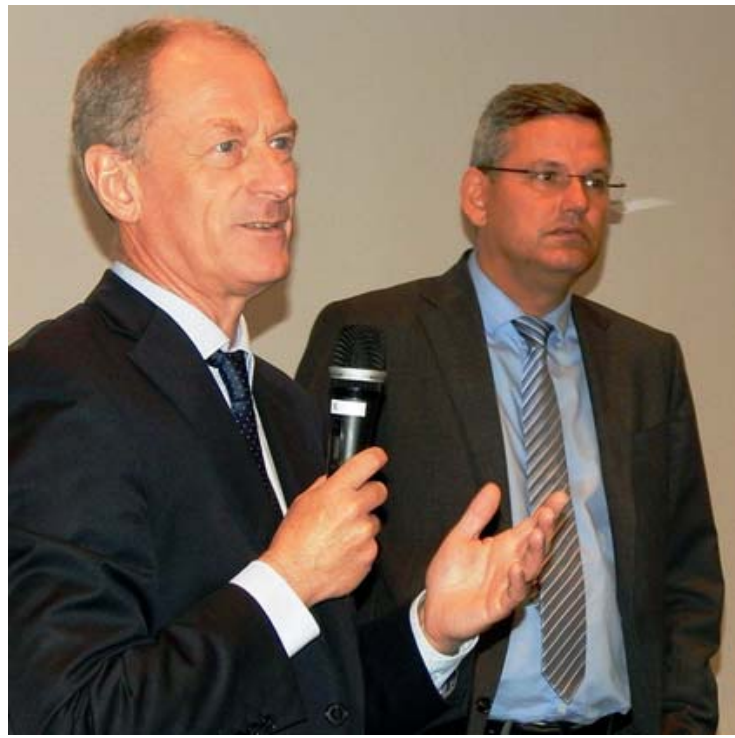

Abb. 4 Ingo Marzi und Wolfgang Böcker diskutieren mit Auditorium. Quelle: AO Foundation

auch wenn geeignete Implantate in Europa bisher noch nicht verfügbar sind.

Am Fersenbein ist aufgrund einer ausgesprochenen Weichteilproblematik eine deutliche Tendenz in Richtung einer minimalinvasiven Platten- oder Schraubenosteosynthese erkennbar. In diesem Sinne stellt auch der Kalkaneusnagel bei ausgewählten Frakturen eine interessante Behandlungsoption dar. Beide Methoden erfordern allerdings eine entsprechende Expertise.

Das 2. Seminar Gießen-Fulda findet am 15. Februar 2019 in Gießen statt.

Prof. Dr. Martin Hessmann, Fulda

Prof. Dr. Christian Heiß, Gießen

Bibliografie

DOI https://doi.org/10.1055/a-0749-4690

OP-JOURNAL 2018; 34: 342-343 ㄷ Georg Thieme Verlag KG Stuttgart · New York ISSN 0178-1715 